Series A

\author{
I. MATHEMATICA
}

494

\title{
BIHARMONIC PROJECTION AND DECOMPOSITION
}

BY

LEO SARIO, CECILIA WANG, and MICHAEL RANGE

HELSINKI 1971

S U O M A L I N EN T I E DEAKATEM I A

doi:10.5186/aasfm.1971.494 
Communicated 12 February 1971 by Oldi Lehto

KESKUSKIRJAPAINO

HELSINKI 1971 


\section{Introduction}

1. The modern theory of harmonic functions owes much of its richness to the striking phenomena encountered in the generalization of the theory from plane regions to abstract Riemann surfaces. In particular, the classification theory and the related compactification theory of Riemann surfaces are among the main achievements in function theory during the past two decades.

In contrast, the theory of biharmonic functions has been restricted mainly to plane regions. One is led to these functions in a natural manner by first replacing $\Delta u=0$ by the equation $\Delta u=$ const.; we call its solutions quasiharmonic. More generally, for any harmonic $h$ one considers $\Delta u=h$, that is, the biharmonic equation $\Delta^{2} u=0$. The reason for the restriction of its theory to plane regions is that the Laplacian is not meaningful on an abstract Riemann surface. However, on a Riemannian manifold the Laplace-Beltrami operator is well-defined, and the theory of quasiharmonic and biharmonic functions can be systematically developed.

This natural observation seems to have far-reaching consequences, and a promising new doctrine is in the making (cf. [2]-[6]). Various boundedness conditions can be imposed separately on the function and its Laplacian. This gives, as it were, a new dimension of richness to the theory of biharmonic functions and the related classification theory of Riemannian manifolds, in comparison with the case of harmonic functions. Analysis on Riemannian manifolds also gains independent raison d'être, not merely as a generalization of a theory developed on Riemann surfaces.

2. The first problem here is to find a biharmonic function $u$ with given boundary values of $u$ and $\Delta u$. We shall solve this problem by what we call the biharmonic projection. The compactification of the manifold, and the boundary on which the values of $u$ and $\Delta u$ are given, will depend on the boundedness properties required of $u$ and $\Delta u$. We shall in fact use simultaneously different compactifications for $u$ and $\Delta u$.

As a by-product we obtain conditions under which a biharmonic function can be decomposed into a harmonic part and a potential part and, as

The work was sponsored by the U. S. Army Research Office-Durham, Grant DA-ARO-D-31-124-71-G20, University of California, Los Angeles. 
a consequence, represented by a pair of harmonic functions. Conversely, we characterize the pairs of harmonic functions which are the harmonic parts and the Laplacians of biharmonic functions.

We shall first consider the case in which the above decomposition requires no conditions on the Riemannian manifold $R$ : functions with positive Laplacians. In other cases a condition on $R$ is unavoidable, but it will suffice to assume that $R \notin O_{Q P}$, that is, $R$ carries positive quasiharmonic functions $u$ with $\Delta u=1$. We shall prove this by establishing the biharmonic projection for functions whose harmonic parts are essentially positive, bounded, Dirichlet finite, or bounded Dirichlet finite, and whose Laplacians possess various boundedness properties.

The present paper includes (but is not restricted to) the proofs and generalizations to unbounded functions of the results announced in NakaiSario [3]. The authors are pleased to acknowledge stimulating discussions with their friend Professor Mitsuru Nakai.

\section{§ 1. The G-operator}

3. On a noncompact Riemannian manifold $R$ of dimension $m \geq 2$ with a smooth metric tensor $\left(g_{i j}\right)$, the Laplace-Beltrami operator is given by

$$
\Delta \cdot=-\frac{1}{\sqrt{g}} \sum_{i=1}^{m} \frac{\partial}{\partial x^{i}} \sum_{j=1}^{m} \sqrt{g} g^{i j} \frac{\partial \cdot}{\partial x^{i}},
$$

where $x=\left(x^{1}, \ldots, x^{m}\right)$ is a local coordinate system, $g=\operatorname{det}\left(g_{i j}\right)$, and $\left(g^{i j}\right)=\left(g_{i j}\right)^{-1}$. We shall assume that $R$ is hyperbolic, i.e., $R \notin O_{G}$. Let $g(x, y)$ be the Green's function on $R$, and $G$ the operator defined by

$$
G f=\int_{R} g(\cdot, y) f(y) d y,
$$

with $d y$ the volume element of $R$. The class

$$
F_{0}=\{f|G| f \mid<\infty\}
$$

will play a fundamental role in the sequel. Given a class $X$ of functions, we set $X_{\Delta}=\{f \mid \Delta f \in X\}$. In particular,

$$
\left(F_{0}\right)_{\Delta}=\left\{f \mid \Delta f \in F_{0}\right\} .
$$

Lemma 1. If $f \in F_{0}$ and $G f \in C^{2}$, then $\Delta G f=f$.

Proof. For every $\varphi \in C_{0}^{\infty}$ we have 


$$
\begin{aligned}
\int_{R} \Delta G f(x) \cdot \varphi(x) d x & =\int_{R} G f(x) \cdot \Delta \varphi(x) d x \\
& =\int_{R}\left[\int_{R} g(x, y) \cdot \Delta \varphi(x) d x\right] f(y) d y \\
& =\int_{R} G \Delta \varphi(y) \cdot f(y) d y=\int_{R} f(y) \cdot \varphi(y) d y .
\end{aligned}
$$

Therefore $\Delta G f=f$ in the sense of distributions, and the lemma follows.

Note that if $f \in F_{0} \cap C^{\infty}$, the above proof and the hypoellipticity of $\Delta$ show that $G f \in C^{\infty}$, hence the lemma applies to such $f$.

4. We refer the reader to Sario-Nakai [7] for general background information needed here and later on harmonizable functions and on Wiener's and Royden's compactifications.

Throughout this paper the term "continuous" will be used in the restricted sense, i.e., with the values $\infty,-\infty$ excluded. We denote by $M_{1}=M_{1}(R)$ the family of continuous harmonizable functions $f$ on $R$ for which there exists a continuous superharmonic function $s_{f}$ with $s_{f} \geq|f|$ on $R$. Let $\tilde{N}_{1}(R)$ be the family of functions in $M_{1}(R)$ which vanish identically on the Wiener harmonic boundary $\delta_{M_{1}}$. Denote by $N_{1}(R)$ the potential subalgebra of $M_{1}(R)$, i.e., the algebra of those functions in $M_{1}(R)$ whose harmonic parts $H_{f}^{R}$ are identically zero on $R$. Clearly $N_{1}(R) \subset \tilde{N}_{1}(R)$. The space $M_{1}(R)$ has the direct sum decompositions

$$
\begin{aligned}
& M_{1}(R)=H P^{\prime}(R) \oplus N_{1}(R), \\
& M_{1}(R)=H B^{\prime}(R) \oplus \tilde{N}_{1}(R),
\end{aligned}
$$

where $H P^{\prime}$ is the class of essentially positive harmonic functions and $H B^{\prime}$ is the class of quasibounded harmonic functions. In general these decompositions are different. For example, on $R=\{z \in C \mid \operatorname{Re} z>0\}$ the function $f(z)=\operatorname{Re} z^{-1}$ is in $H P^{\prime}(R)$ but not in $H B^{\prime}(R)$.

When no ambiguity can arise, we will often omit the symbol $(R)$ in our notation.

Lemma 2. If $f \in F_{0}$ and $G f$ is continuous, then $G f \in N_{1}(R)$.

Proof. Let $f=f^{+}-f^{-}$with $f^{+}=f \cup 0$ and $f^{-}=(-f) \cup 0$. Since $f \in F_{0}, G f^{+}$and $G f^{-}$are well defined and $G f=G f^{+}-G f^{-}$. By the super mean value property and the lower semicontinuity we see that $G f^{+}$and $G f^{-}$are superharmonic, hence harmonizable, and $|G f| \leq G f^{+}+G f^{-}$. Therefore $G f \in M_{1}(R)$, and it suffices to show that $H_{G f}=0$.

Suppose that for a given superharmonic function $s$ on $R$ there exists a subharmonic function $v$ such that $s \geq v$ on $R$. Then by the Riesz 
representation theorem there exists a unique harmonic function $h_{s}$ and a unique measure $\sigma_{s}$ on $R$ such that

$$
s=h_{s}+\int_{R} g(\cdot, y) d \sigma_{s}(y),
$$

with $h_{s}$ the greatest harmonic minorant G.H.M. s. In particular,

$$
G f^{+}=h_{G f}+\int_{R} g(\cdot, y) d \sigma_{G f}+(y),
$$

and we conclude by

$$
G f^{+}=\int_{R} g(\cdot, y) f^{+}(y) d y
$$

and the uniqueness of the representation that G.H.M. $G f^{+}=0$.

On a regular subregion $\Omega$, we have $G f^{+}=H_{G f^{+}}^{\Omega}+p^{\Omega}$, where $H_{G f^{+}}^{\Omega}$ is the harmonic function on $\Omega$ with boundary values $G f^{+}$on $\partial \Omega$. Clearly $0 \leq H_{G f+}^{\Omega}$ $\leq G f^{+}$on $\Omega$. On taking the limit $\Omega \rightarrow R$ we obtain $0 \leq H_{G f^{+}}^{R} \leq G f^{+}$. Since G.H.M. $G f^{+}=0$, it follows that $H_{G f^{+}}^{R}=0$, that is, $G f^{-} \in N_{1}(R)$. The same holds for $G f^{-}$, and thus $G f \in N_{1}(R)$.

\section{§ 2. Functions with positive Laplacians}

5. Denote by $W(R)$ the family of biharmonic functions, that is, $C^{\mathbf{4}_{-}}$ functions satisfying $\Delta^{2} u=0$ on $R$. Let $H, B, P, D$ be the classes of harmonic functions, bounded functions, nonnegative functions, and functions with finite Dirichlet integrals. We will be interested in the subfamilies $W X Y_{\Delta}=W \cap X \cap Y_{\Delta}$ of $W$, with $X, Y=B, P$, or $D$. It will be illuminating to first consider $W K S S_{\Delta}$, where

$$
S=\left\{f \in C^{2} \mid \Delta f \geq 0\right\}
$$

and $K$ denotes the class of harmonizable functions.

Theorem 1. On an arbitrary hyperbolic Riemannian manifold the functions in $K S S_{\Delta}$ have a unique decomposition into biharmonic functions and potentials:

$$
K S S_{\Delta}=W K S S_{\Delta} \oplus N_{1}\left(N_{1}\right)_{\Delta} S S_{\Delta} .
$$

Proof. We will show first that every harmonizable function $f \in S$ has a Riesz decomposition

where $\Delta f \in F_{0}$.

$$
f=H_{f}+G \Delta f,
$$


Let $\Omega$ be a regular subregion of $R$ and $g_{\Omega}(x, y)$ the Green's function on $\Omega$. For a ball $B_{x}$ about $x \in \Omega$ with radius $\varepsilon$ such that $\bar{B}_{x} \subset \Omega$, Green's formula yields

$$
\begin{aligned}
& \int_{\partial\left(\Omega-B_{x}\right)}\left\{g_{\Omega}(x, y) * d\left[f(y)-H_{f}^{\Omega}(y)\right]-\left[f(y)-H_{f}^{\Omega}(y)\right] * d_{y} g_{\Omega}(x, y)\right\} \\
= & -\int_{\Omega-B_{x}}\left\{g_{\Omega}(x, y) \Delta\left[f(y)-H_{f}^{\Omega}(y)\right]-\left[f(y)-H_{f}^{\Omega}(y)\right] \Delta_{y} g_{\Omega}(x, y)\right\} d y,
\end{aligned}
$$

where $H_{f}^{\Omega}$ is the harmonic function on $\Omega$ with boundary values $f$ on $\partial \Omega$. On letting $\varepsilon \rightarrow 0$ we obtain

$$
f(x)=H_{f}^{\Omega}(x)+\int_{\Omega} g_{\Omega}(x, y) \Delta f(y) d y .
$$

Since $f$ is harmonizable, $H_{f}^{\Omega}(x)$ tends to a harmonic function $H_{f}(x)$ on $R$ as $\Omega \rightarrow R$. The monotone convergence theorem now gives the desired result.

Let $f \in K S S_{\Delta}$. Then also $\Delta f \in K S$ because $\Delta f \geq 0$ and $\Delta^{2} f \geq 0$, that is, $\Delta f$ is superharmonic. Hence we have

$$
\Delta f=H_{\Delta f}+G \Delta^{2} f \text {. }
$$

Clearly $0 \leq H_{\Delta f} \leq \Delta f$, and thus $H_{\Delta f} \in F_{0}$. We therefore obtain

$$
f=H_{f}+G \Delta f=\left(H_{f}+G H_{\Delta f}\right)+\left(G \Delta f-G H_{\Delta f}\right) .
$$

By means of Lemmas 1 and 2 it is now easy to see that $\left(H_{f}+G H_{\Delta f}\right) \in W K S S_{\Delta}$ and $\left(G \Delta f-G H_{\Delta f}\right) \in N_{1}\left(N_{1}\right)_{\Delta} S S_{\Delta}$.

To prove the uniqueness of the decomposition, let $f \in W K S S_{\Delta} \cap$ $N_{1}\left(N_{1}\right)_{\Delta}$. Since $\Delta f \in H P \cap N_{1}, \Delta f \equiv 0$ on $R$. Thus $f \in H \cap N_{1}$, and we conclude that $f \equiv 0$ on $R$.

This completes the proof.

We call $W_{K S S_{\Delta}}$ the biharmonic projection of $K S S_{\Delta}$. Note that we have, in particular, the above decomposition for positive functions in $S S_{\Delta}$, since $P S \subset K$.

6. On observing that $W S S_{\Delta}(R)=W S(R)=W P_{\Delta}(R)$, we obtain the following Riesz decomposition for $W K P_{\Delta}(R)$ :

Theorem 2. On an arbitrary hyperbolic Riemannian manifold every harmonizable biharmonic function with a positive Laplacian has a unique decomposition into a harmonic function and the potential of a positive harmonic function,

$$
w=u+G v, w \in W K P_{\Delta}, u \in H, v \in H P .
$$


Every function $w$ which has such a decomposition is harmonizable, hence we cannot suppress this assumption. For example, the function $f(z)=-|z|^{2}$ on $R=\{z \in C|| z \mid>1\}$ is in $W P_{\Delta}$, but not harmonizable.

For any Riesz decomposition $w=u+G v, w \in W X Y_{\Delta}, \quad u \in H X$, $v \in H Y$, we call $u$ the harmonic part and $G v$ the potential part of $w$.

\section{§ 3. Function classes}

7. Theorems 1 and 2 state that $K S S_{\Delta}(R)$ has the biharmonic projection and $W K P_{\Delta}$ has the Riesz decomposition with no restrictions on the functions in $K S S_{\Delta}(R)$ or on the manifold $R \notin O_{G}$. However, this is not true in general (see Nakai-Sario [5]). In the remainder of this paper, we shall establish conditions under which it is possible to have biharmonic projections and Riesz decompositions for certain classes of functions which we now introduce.

Let $M_{2}(R)$ and $N_{2}(R)$ be the Wiener algebra and the Wiener potential subalgebra (see e.g. Sario-Nakai [7]). It is clear that $M_{2}(R)=M_{1}(R) \cap B(R)$ and $N_{2}(R)=N_{1}(R) \cap M_{2}(R)$. Denote by $M_{3}(R)$ the class of Tonelli functions with finite Dirichlet integrals and by $N_{3}(R)$ the subclass of functions $f \in M_{3}(R)$ which vanish on the Royden harmonic boundary $\delta_{M_{3}}$. It is known that $M_{3}(R) \subset M_{1}(R)$ and that $N_{3}(R)=N_{1}(R) \cap M_{3}(R)$ (see Constantinescu-Cornea [1]), hence the subclasses of $M_{3}(R)$ corresponding to $N_{1}(R)$ and $\tilde{N}_{1}(R)$ coincide. Clearly the Royden algebra $M_{4}(R)=M_{3}(R) \cap B(R)$ and the Royden potential subalgebra $N_{4}(R)=$ $N_{3}(R) \cap M_{4}(R)=N_{1}(R) \cap M_{4}(R)$. For simplicity, we write $M_{i j}(R)=$ $M_{i}(R) \cap\left(M_{j}\right)_{\Delta}(R)$ and $N_{i j}(R)=N_{i}(R) \cap\left(N_{j}\right)_{\Delta}(R), \quad i, j=1,2,3,4$.

8. The potential subalgebras $N_{i}(R)$ have the following property:

Lemma 3. If $f \in\left(F_{0}\right)_{\Delta} \cap N_{i}(R)$ for $i=1,2,3,4$, then $G \Delta f=f$.

Proof. We have shown that for any regular subregion $\Omega$ of $R$,

$$
f(x)=H_{f}^{\Omega}(x)+\int_{\Omega} g_{\Omega}(x, y) \Delta f(y) d y .
$$

Since $f \in N_{i}(R), \lim _{\Omega \rightarrow R} H_{f}^{\Omega}(x)=0$ on $R$. By Lebesgue's dominated convergence theorem we conclude that

$$
f(x)=\int_{R} g(x, y) \Delta f(y) d y
$$

on $R$. 


\section{$\S 4$. Funetions with essentially positive harmonic parts}

9. Set $\Phi_{1 j}=M_{1 j}\left(F_{0}\right)_{\tau \Delta}, j=1,2,3,4$, where $\pi$ stands for the harmonic projection. Clearly $W \Phi_{1 j}=W M_{1 j}\left(F_{0}\right)_{\Delta}$.

Theorem 3. The direct sum decomposition

$$
\Phi_{1_{j}}=W \Phi_{1_{j}} \oplus N_{1_{j}} \Phi_{1_{j}},
$$

$j=1,2,3,4$, is valid on an arbitrary hyperbolic Riemannian manifold.

Proof. Let $f \in \Phi_{11}$. By the direct sum decomposition $M_{1}(R)=H P^{\prime}(R)$ $\oplus N_{1}(R), f=\pi f+k$ with $\pi f \in H P^{\prime}, k \in N_{1}$, and $\Delta k=\Delta f$. Since $\Delta f \in M_{1}$ we obtain $\Delta f=\pi \Delta f+p$ with $\pi \Delta f \in F_{0}, p \in N_{1}$. Therefore $f=\pi f+k=$ $(\pi f+G \pi \Delta f)+(k-G \pi \Delta f)$, and one verifies immediately that $(\pi f+$ $G \pi \Delta f) \in W \Phi_{11}$ and $(k-G \pi \Delta f) \in N_{11} \Phi_{11}$.

The uniqueness is clear, and we have proved the theorem for $\Phi_{11}$.

It is known that $M_{2}(R)=H B(R) \oplus N_{2}(R), M_{3}(R)=H D(R) \oplus N_{3}(R)$, and $M_{4}(R)=H B D(R) \oplus N_{4}(R)$. By means of these decompositions the theorem follows for $\Phi_{1 j}, j=2,3,4$, in analogy with the proof above.

10. In view of Lemma 2 we have also proved:

Theorem 4. On an arbitrary hyperbolic Riemannian manifold, the functions $w_{j} \in W \Phi_{1 j}$ have the unique decompositions $u_{j}+G v_{j}$ with

$$
\begin{aligned}
& u_{1} \in H P^{\prime}, v_{1} \in H P^{\prime}, \\
& u_{2} \in H P^{\prime}, v_{2} \in H B, \\
& u_{3} \in H P^{\prime}, v_{3} \in H D, \\
& u_{4} \in H P^{\prime}, v_{4} \in H C,
\end{aligned}
$$

where $C$ stands for $B D$.

11. We proceed to a more detailed study of the cases $j=2,4$. By imposing a simple condition on $R$ we will be able to dispense with the $\left(F_{0}\right)_{\pi \Delta}$-requirement.

We call a $C^{2}$-function $u$ on $R$ with $\Delta u \equiv 1$ quasiharmonic and denote by $Q(R)$ the class of these functions. For a given class $X$ of functions let $O_{X}$ be the class of Riemannian manifolds $R$ on which there exist no nonconstant $X$-functions. It is known that $R \notin O_{Q P}$ if and only if $G 1<\infty$ (see Nakai-Sario [3], [4]). Thus if $R \notin O_{Q P}$, then $\Phi_{12}=M_{12}$ and $\Phi_{14}=$ $M_{14}$.

Lemma 4. If $R \notin O_{Q P}$, then $f \in C^{2} \cap B_{\Delta}$ implies that $f$ is harmonizable on $R$. 
Proof. Without loss of generality we may assume that $|\Delta f| \leq 1$. We know that on a regular region $\Omega$ of $R, f(x)=H_{f}^{\Omega}(x)+\int_{\Omega} g_{\Omega}(x, y) \Delta f(y) d y$. In view of $\left|g_{\Omega} \Delta f\right|<g$ and $\int_{R} g d y<\infty \quad$ we conclude by Lebesgue's theorem that $\int_{\Omega} g_{\Omega} \Delta f d y$ in converges as $\Omega \rightarrow R$. Therefore $H_{f}^{\Omega}$ converges and $f$ is harmonizable.

Theorem 5. If $R \notin O_{Q P}$, then

$$
\begin{aligned}
& M_{12}(R)=W M_{1} B_{\Delta}(R) \oplus N_{12}(R), \\
& M_{14}(R)=W M_{1} C_{\Delta}(R) \oplus N_{14}(R),
\end{aligned}
$$

and

$$
\begin{aligned}
& W M_{1} B_{\Delta}(R) \subset H P^{\prime}(R) \oplus G H B(R), \\
& W M_{1} C_{\Delta}(R) \subset H P^{\prime}(R) \oplus G H C(R) .
\end{aligned}
$$

12. Theorem 5 gives the linear mappings

$$
\begin{aligned}
& \gamma_{12}: W M_{1} B_{\Delta} \rightarrow H P^{\prime} \times H B, \\
& \gamma_{14}: W M_{1} C_{\Delta} \rightarrow H P^{\prime} \times H C .
\end{aligned}
$$

The solvability of the biharmonic Dirichlet problem depends on the surjectivity of these mappings. This in turn can be completely characterized:

Theorem 6. $W M_{1} B_{\Delta} \cong H P^{\prime} \times H B$, and also $W M_{1} C_{\Delta} \cong H P^{\prime} \times H C$, if and only if $R \notin O_{Q P}$.

Proof. On $R \notin O_{Q P}$, let $u_{1} \in H P^{\prime}$ and $u_{2} \in H B$ be given. Clearly $G u_{2}$ is well defined and, by Lemma $1, \Delta G u_{2}=u_{2}$. Lemma 4 gives $u_{1}+$ $G u_{2} \in W M_{1} B_{4}$ and therefore $W M_{1} B_{4} \cong H P^{\prime} \times H B$.

Conversely, $\quad \gamma_{12}^{-1}(0,1)=\int_{R} g(\cdot, y) d y \in Q P$, and a fortiori $R \notin O_{Q P}$.

The proof of $W M_{1} C_{\Delta} \cong H P^{\prime} \times H C$ is similar.

\section{§ 5. Functions with bounded harmonic parts}

13. Let

$$
\begin{gathered}
F_{1}=\left\{f \in F_{0}\left|\sup _{x \in R}\right| G f(x) \mid<\infty\right\}, \\
\Phi_{2 j}=M_{2 j}\left(F_{1}\right)_{\tau \Delta},
\end{gathered}
$$

$j=1,2,3,4$. 
Theorem 7. On an arbitrary hyperbolic Riemannian manifold,

$$
\Phi_{2 j}=W \Phi_{2 j} \oplus N_{2 j} \Phi_{2 j} .
$$

Moreover, the functions $w_{j} \in W \Phi_{2 j}$ have the unique decompositions $u_{j}+$ $G v_{j}$ with

$$
\begin{array}{ll}
u_{1} \in H B, & v_{1} \in H P^{\prime}, \\
u_{2} \in H B, & v_{2} \in H B, \\
u_{3} \in H B, & v_{3} \in H D, \\
u_{4} \in H B, & v_{4} \in H C .
\end{array}
$$

The proof is similar to those of Theorems 3 and 4. The assumption $\left(F_{1}\right)_{\pi \Delta}$ is to assure the boundedness of the potential part of the biharmonic projection $W \Phi_{2 j}$.

It is known that $O_{Q P}<O_{Q B}$ and that $R \notin O_{Q B}$ if and only if $\sup _{R}|G 1|<\infty \quad$ (Nakai-Sario [4]).

Theorem 8. If $R \notin O_{Q B}$, then

$$
\begin{aligned}
& M_{22}(R)=W B B_{\Delta}(R) \oplus N_{22}(R), \\
& M_{24}(R)=W B C_{\Delta}(R) \oplus N_{24}(R) .
\end{aligned}
$$

Furthermore, if $R \notin O_{Q P}$, then

$$
\begin{aligned}
& W B B_{\Delta}(R) \subset H B(R) \oplus G H B(R), \\
& W B C_{\Delta}(R) \subset H B(R) \oplus G H C(R) .
\end{aligned}
$$

Theorem 9. $W B B_{\Delta} \cong H B \times H B$, and also $W B C_{\Delta} \cong H B \times H C$, if and only if $R \notin O_{Q B}$.

\section{§ 6. Dirichlet integrals}

14. Let

$$
F_{2}=\left\{f \in F_{0} \mid G(|f|,|f|)<\infty\right\},
$$

where

$$
G\left(f_{1}, f_{2}\right)=\int_{R \times R} g(x, y) f_{1}(x) f_{2}(y) d x d y .
$$

We denote the Dirichlet integral of $f$ by $D(f)$.

Lemma 5. If $f \in F_{2} \cap C^{\infty}$, then $D(G f)=G(f, f)$. 
Proof. Let $\Omega$ be a regular subregion of $R$ and $g_{\Omega}(x, y)$ the Green's function on $\Omega$. Define $g_{\Omega}(x, y)=0$ for $x$ or $y \in R-\Omega$ and set $G_{\Omega} f(x)=$ $\int_{R} g_{\Omega}(x, y) f(y) d y$. Then Stokes' formula yields

$$
\begin{aligned}
0 & =\int_{\partial \Omega} G_{\Omega} f(x) * d G_{\Omega} f(x) \\
& =D_{R}\left(G_{\Omega} f\right)-\int_{R} G_{\Omega} f(x) \Delta G_{\Omega} f(x) d x .
\end{aligned}
$$

Since $\Delta G_{\Omega} f=f$ on $\Omega$,

$$
\begin{aligned}
D_{R}\left(G_{\Omega} f\right) & =\int_{R} G_{\Omega} f(x) f(x) d x \\
& =\int_{R \times R} g_{\Omega}(x, y) f(x) f(y) d x d y \\
& \leq G(|f|,|f|)<\infty .
\end{aligned}
$$

By Lebesgue's theorem

$$
G f(x)=\lim _{\Omega \rightarrow R} G_{\Omega} f(x),
$$

and by Fatou's lemma,

$$
\begin{aligned}
D_{R}(G f) & \leq \liminf _{\Omega \rightarrow R} D_{R}\left(G_{\Omega} f\right) \\
& \leq G(|f|,|f|)<\infty .
\end{aligned}
$$

A fortiori Lebesgue's theorem gives

$$
\begin{aligned}
D_{R}(G f) & =\lim _{\Omega \rightarrow R} D_{R}\left(G_{\Omega} f\right) \\
& =\lim _{\Omega \rightarrow R} \int_{R \times R} g_{\Omega}(x, y) f(x) f(y) d x d y \\
& =\int_{R \times R} g(x, y) f(x) f(y) d x d y \\
& =G(f, f) .
\end{aligned}
$$

The proof is herewith complete. 


\section{§ 7. Functions with Dirichlet finite harmonic parts}

15. Consider

$$
\Phi_{3 j}=M_{3 j}\left(F_{2}\right)_{\pi \Delta}
$$

with $j=1,2,3,4$.

Theorem 10. On an arbitrary hyperbolic Riemannian manifold,

$$
\Phi_{3 j}=W \Phi_{3 j} \oplus N_{3 j} \Phi_{3 j} .
$$

Proof. Let $f \in \Phi_{31}$. As in the proof of Theorem 3, we can write

$$
f=(\pi f+G \pi \Delta f)+(k-G \pi \Delta f)
$$

with $\pi f \in H D, k \in N_{3}$, and $\pi \Delta f \in F_{2} \cap C^{\infty}$. Using Lemma 5 we obtain $D(G \pi \Delta f)<\infty$, and thus also $D(k-G \pi \Delta f)<\infty$. It is now clear that the above decomposition is the desired one and that it is unique.

The proofs for the other classes are similar.

Theorem 11. On an arbitrary hyperbolic Riemannian manifold, the functions $w_{j} \in W \Phi_{3 j}$ have the unique decompositions $u_{j}+G v_{j}$ with

$$
\begin{aligned}
& u_{1} \in H D, \quad v_{1} \in H P^{\prime}, \\
& u_{2} \in H D, \quad v_{2} \in H B, \\
& u_{3} \in H D, \quad v_{3} \in H D, \\
& u_{4} \in H D, \quad v_{4} \in H C .
\end{aligned}
$$

16. It is known that $O_{Q P}<O_{Q D}$ and that $R \notin O_{Q D}$ if and only if $G(1,1)<\infty \quad$ (Nakai-Sario [4]).

Theorem 12. If $R \notin O_{Q D}$, then

$$
\begin{aligned}
& M_{32}(R)=W D B_{\Delta}(R) \oplus N_{32}(R) \\
& M_{34}(R)=W D C_{\Delta}(R) \oplus N_{34}(R) .
\end{aligned}
$$

Furthermore, if $R \notin O_{Q P}$, then

$$
\begin{aligned}
& W D B_{\Delta}(R) \subset H D(R) \oplus G H B(R) \\
& W D C_{\Delta}(R) \subset H D(R) \oplus G H C(R) .
\end{aligned}
$$

Theorem 13. $W D B_{\Delta} \cong H D \times H B$, and also $W D C_{\Delta} \cong H D \times H C$, if and only if $R \notin O_{Q D}$. 


\section{§ 8. Functions with bounded Dirichlet finite harmonic parts}

17. We close by considering the class

$$
\Phi_{4 j}=M_{4 j}\left(F_{1}\right)_{\pi \Delta}\left(F_{2}\right)_{\pi \Delta},
$$

with $j=1,2,3,4$.

Theorem 14. On an arbitrary hyperbolic Riemannian manifold,

$$
\Phi_{4 j}=W \Phi_{4 j} \oplus N_{4 j} \Phi_{4 j}
$$

Moreover, the functions $w_{j} \in W \Phi_{4 j}$ have the unique decompositions $u_{j}+G v_{j}$ with

$$
\begin{aligned}
& u_{1} \in H C, \quad v_{1} \in H P^{\prime}, \\
& u_{2} \in H C, \quad v_{2} \in H B, \\
& u_{3} \in H C, \quad v_{3} \in H D, \\
& u_{4} \in H C, \quad v_{4} \in H C .
\end{aligned}
$$

18. It is known that $O_{Q C}=O_{Q B} \cup O_{Q D}$ and that $R \notin O_{Q C}$ if and only if $\sup _{R}|G 1|<\infty$ and $G(1,1)<\infty$ (Nakai-Sario [4]).

Theorem 15. If $R \notin O_{Q C}$, then

$$
\begin{aligned}
& M_{42}(R)=W C B_{\Delta}(R) \oplus N_{42}(R), \\
& M_{44}(R)=W C C_{\Delta}(R) \oplus N_{44}(R) .
\end{aligned}
$$

If $R \notin O_{Q P}$, then

$$
\begin{aligned}
& W C B_{\Delta}(R) \subset H C(R) \oplus G H B(R), \\
& W C C_{\Delta}(R) \subset H C(R) \oplus G H C(R) .
\end{aligned}
$$

Theorem 16. $W C B_{\Delta} \cong H C \times H B$, and also $W C C_{\Delta} \cong H C \times H C$, if and only if $R \notin O_{Q C}$.

University of California

Los Angeles, Cal., U.S.A. 


\section{References}

[1] Constantinescu, C. - Connea, A., Ideale Ränder Riemannscher Flächen, Springer, 1963, $244 \mathrm{pp}$.

[2] Kwon, Y. K. - Sario, L. - Walsh, B., Behavior of biharmonic functions on Wiener's and Royden's compactifications, Ann. Inst. Fourier (Grenoble) (to appear).

[3] Nakai, M. - Sario, L., Biharmonic classification of Riemannian manifolds, Bull. Amer. Math. Soc. 77 (1971), 432-436.

[4] -»- - - Quasiharmonic classification of Riemannian manifolds, Proc. Amer. Math. Soc. (to appear).

[5] -»- - - - A property of biharmonic functions with Dirichlet finite Laplacians, Math. Scand. (to appear).

[6] -»- - - Dirichlet finite biharmonic functions with Dirichlet finite Laplacians, Math. Z. (to appear).

[7] Sario, L. - NakaI, M., Classification theory of Riemann surfaces, Springer, 1970, $446 \mathrm{pp}$. 